\title{
Wikipédia As A Learning Tool
}

\author{
Büttenbender, M.D. ${ }^{1}$; Rocha, C.E.S. ${ }^{1}$; Denardin, E.L.G. ${ }^{2}$, Roehrs, R. ${ }^{1,2}$ \\ ${ }^{1}$ Grupo Interdisciplinar de Pesquisa em Prática de Ensino, Unipampa, RS, Brazil \\ ${ }^{2}$ Laboratório de Estudos Físico-Químicos e Produtos Naturais, Unipampa, RS, Brazil
}

INTRODUCTION: It is common to use websites as references for research in school settings. Wikipedia emerged in the mid-2000s promoting a big change compared to other encyclopedias, mainly because it is in digital form and allow its users to create and edit information contained therein. Due to its easiness of use and readiness, it gained prominence as a research resource and tool. By doing research on biochemistry using Wikipedia, we can see a vast amount of content that assists in learning, however giving space for questions recurring about its reliability. OBJECTIVES: The research aims to verify if the information about Biochemistry found on Wikipedia are consistent to those found in the book "Lehninger Principles of Biochemistry" and present its references. MATERIAL AND METHODS: In order to evaluate selected topics in the biochemistry such as proteins, carbohydrates, lipids and nucleic acids; the rates of Wikipedia and the book were compared and also the concepts presented in each subject in order to analyze the approach and content of information that the Wiki uses. RESULTS AND DISCUSSION: Considering only the indices, it is possible to say that the contents found in the Wiki list the same issues that the book addresses, but organization is different. It also features several references from books of Biochemistry. When comparing the information contained in Wikipedia, we realize that they are in accordance with those found in the book, but in a superficial way, making new sources of knowledge necessary to delve into the content. CONCLUSIONS: Wikipedia can and should be used as an introductory tool for acquiring knowledge about biochemistry. However, like any source of consultation, the Wiki is not able to replace all other sources of reference since its approaches are brief, making the studies easily conducted and reaching only superficial levels.

Keywords: Wikipedia, search, biochemistry. 